\title{
Sdn for Load Balancing Nodes in Wireless Sensor Network
}

\section{Archana, S Prakasam}

\begin{abstract}
In this paper, we study about Software Defined Networking (SDN) is centralized controller of the network. It has maintained the information regarding the whole network which being able to optimize the available network resources and makes the route decision for forward packets. In Traditional networks, each device possess with own configuration and state which makes difficult to networking and expensive to maintain and forward packets. It also uses multiple routing algorithms for many different devices such as routers and switches and no centralized approach for routing. SDN architecture is centralized and support different routing algorithms for packet forwarding in emerging changes in networks. SDN usage is rapidly increased in networking to meet today challenges. This paper discuss on SDN with Wireless Sensor Network for load balancing between the nodes in communication. Due to Mobility of node in Wireless sensor network need of load balance. Also discussed various issues related with SDN performance in wireless sensor Network.

Keywords: Software defined network, Open Flow, Network State
\end{abstract} Information, Wireless Sensor Network, SDN-WISE, SD-WSN.

\section{INTRODUCTION}

A wireless sensor network (WSN) is a collection of sensor nodes to record the physical condition of environment such as temperature, pressure etc. and to collect the information. Information passes in the network to a centralized node as sink node. WSN used in various fields such as agriculture, forest, road traffic and health issue for information collection. In WSN there is a limitation for increase in energy usage due to battery power and delay in information transmission due to mobility. To overcome limitation a load balancing approach for communication of sensor network is possible through Software defined network [1]. Software defined networking is a new paradigm in network to manage control and data plane easier .SDN is an open source to control and manage network elements. Traditional network devices makes complex in control and management on each device [2].SDN separates the control plane and data plane in network layer. SDN controller is central which separate routers and switches to make a forwarding devices simple.SDN provide automated load balancing, routing and reduced hardware costs. SDN supports Protocols such as Open flow, open stack, open daylight controller to make route decision in forwarding packets [3]. Open Flow [4] protocol at southbound interface which is interface with network infrastructure as wired or wireless devices

Revised Manuscript Received on June 15, 2020.

* Correspondence Author

D. Archana*, Assistant Professor, Department of Computer Science, Arcot Sri Mahalakshmi Women's College, Villapakkam, Ranipet, Tamil Nadu, India.

Dr. S. Prakasam, Associate Professor, Department of Computer Science and Applications Department, Sri Chandrasekharendra Saraswathi Viswa Mahavidyalaya (SCSVMV) University, Enathur, Kanchipuram, Tamil Nadu, India.

(C) The Authors. Published by Blue Eyes Intelligence Engineering and Sciences Publication (BEIESP). This is an open access article under the CC BY-NC-ND license (http://creativecommons.org/licenses/by-nc$\underline{\mathrm{nd} / 4.0 /)}$
SDN with WSN impose more challenges as limited in energy consumption, memory, processing resources and application related to nodes performance. This work consists of following section. Section II related works. Section III describes the SD-WSN. Section IV load balancing in networking using SDN.SDN avoid delay in communication using these approach. Section V concludes this work.

\section{RELATED WORK}

WSN is used various fields such as agriculture monitoring, weather monitoring, health monitoring, industrial monitoring. Nowadays a variety of uses of WSN with low energy and power consumption.WSN have challenges for resource constraints such as energy, processing, memory communication. Nodes in WSN have mobility [5] [6].

SDN with WSN many researchers have been taken place for efficient use of energy in network. In this network lifetime also increased for nodes and reduce the network, transmission cost [7].SDN for wireless network need to broadcast information, to find mobility of node, hidden node, node status. Open Flow protocol has to uninterrupted data collection from node in wireless network. Node will be in active state or passive state. Open flow protocol with flow sensor used in WSN [8].

Many research is done on SDN but only few research is takes place in SDN ,WSN and security in future effort that takes security into consideration[9].Research challenges in SDN-WSN is energy efficient, routing, mobility, reliability, QOS, management, localization, wireless power transmission, security[10].

\section{SD -WSN}

\section{A. SDN ARCHITECTURE}

Software-Defined Networking (SDN) is a network which separates the Control plane from the data plane and centralizes the controller. The goal of SDN is to provide open, user controlled, managing and network elements. The data plane in SDN is responsible for packet forwarding hardware which is not possible in traditional networks [11]. In Fig 1 show the SDN Architecture that separates data plane with control plane. Software defined networking defines with three layers: Control, Data and Application: 


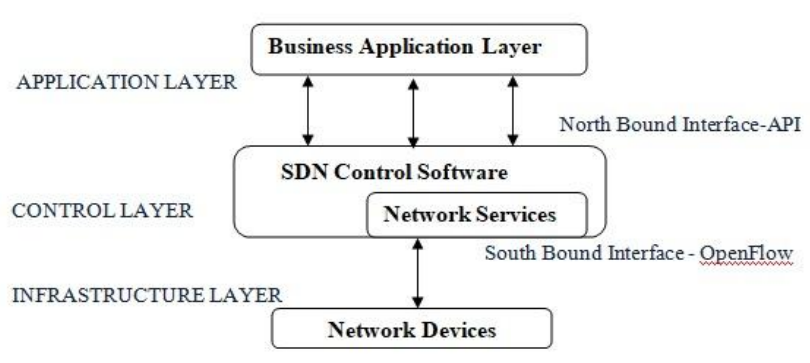

Fig 1. SDN Architecture.

1) Control plane: The control plane carries traffic signal and responsible for routing in network. The source to destination transmission is takes place by control plane. The function of control plane is to make Centralized configuration and manage [12] .The application layer sends instruction to SDN control software from various business Application layer. The control layer provides data to networking elements. Vice versa the control layer separates information from the networking elements and sends back to application layer. The SDN controller controls the different network devices for routing and switching by separating data plane and control plane. Various protocols used for routing like OSPF, RIP that are managed by control plane

2) Data Plane: The data plane is used only for forwarding the packets using different protocols. Traffic is handling by control plane while transmission.

3) Application Plane: Application layer is a collection of different application to perform the task. The application layer send data to SDN controller through north bound interface to perform the function.

Need For SDN:

$>$ Flow Optimize.

$>$ Virtual router.

$>$ Load Balancer.

$>$ Protector.

$>$ Flow Steering

SDN is virtual software to control network devices. So no need to change the device, in place change the software used in routers and switches to manage the traffic. This external is SDN Controller or Network Operating System. It obtains the network state information and makes necessary decision for forwarding the packets. Several new applications develop to control the traffic and delay in transmission in SDN controller makes easy. The SDN Controller makes rules in flow table send to data plane for forwarding the packets. The SDN support different Controllers Such as Open Daylight, Flood light, ONOS, open switch [13].

\section{B. Open Daylight:}

The Open Daylight project is a build on open source Linux software. It provides centralized, programmatic control and device monitoring. Open daylight provides an interface to infrastructure layer through open flow to perform networking in efficient manner. Open day light protocol work on hardware that supports the Java platform [14].
$>$ Network advisor.

Open day light protocol lies at northbound interface to communicate with application layer and send information to the controller to forward the packets. This supports various network environments and SDN Controller.

\section{Open Network Operating System (ONOS)}

ONOS is an open source operating system developed at ON lab. This provides scalability, efficient performance and high availability for communication. It performs network operation with easy programming interface. This OS provides High level of abstraction in application which makes to know about the state of the network and traffic in network. ONOS provides network graph through which get topology of the network. ONOS manages and control the entire network, simplifies to deploy new software, hardware and services. ONOS supports multiple servers and run as distributed system [15].

The SDN controller performs three functions:

i) Collect Network State Information: In Fig 2 shows SDN controller first collects the network state information globally and then makes routing decision to compute new path. The topology control is classified as static and dynamic link. In Static link topology not change so routing decision is simple but in dynamic link topology continuous change so need to collect the network state at every intervals. This faces many challenges in dynamic link to collect accurate network state information which help to utilize the network resources and avoid congestion problem [16].

ii) Compute feasible shortest paths: After obtaining network state information and discovering topology, it obtains the optimal path for routing the packets using algorithms.

iii) Forwarding Protocols: The SDN controller use Open Flow protocol to forward the packets. The forwarding table is maintaining for each switches.

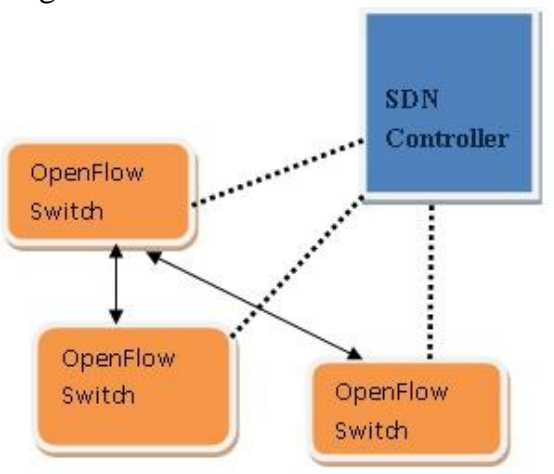

Fig 2 Centralized Controllers

The Different Routing Algorithms is used to forward the packets based on Open Flow Protocols. The existing routing Algorithms based on either Static link or Dynamic Link in network. The various algorithms designed to collect network state information for static link and dynamic link. The dynamic link must need to update flow table for forwarding the packets at continuous interval but static link no need to continuous update of flow table.

Published By:

Blue Eyes Intelligence Engineering 
So the routing algorithm is selected based on which perform efficient to collect accurate network state information. Network state information is obtained from SDN switches by querying them. Hence the future algorithms are needed to take probabilistic metrics to find accurate network state information [17] [18].

\section{Wireless Sensor Network with SDN Architecture}

WSN is a distributed sensor nodes to sense and control the node in network. Each node in WSN is communicated with other node within the range. Each range has a sink node which communicates with other network node. The control of nodes in WSN is classified as centralized, decentralized or distributed control. It is difficult to manage node due to scattered of nodes. Another challenge is high energy consumption, mobility of nodes, node failures and battery power [19] [20].

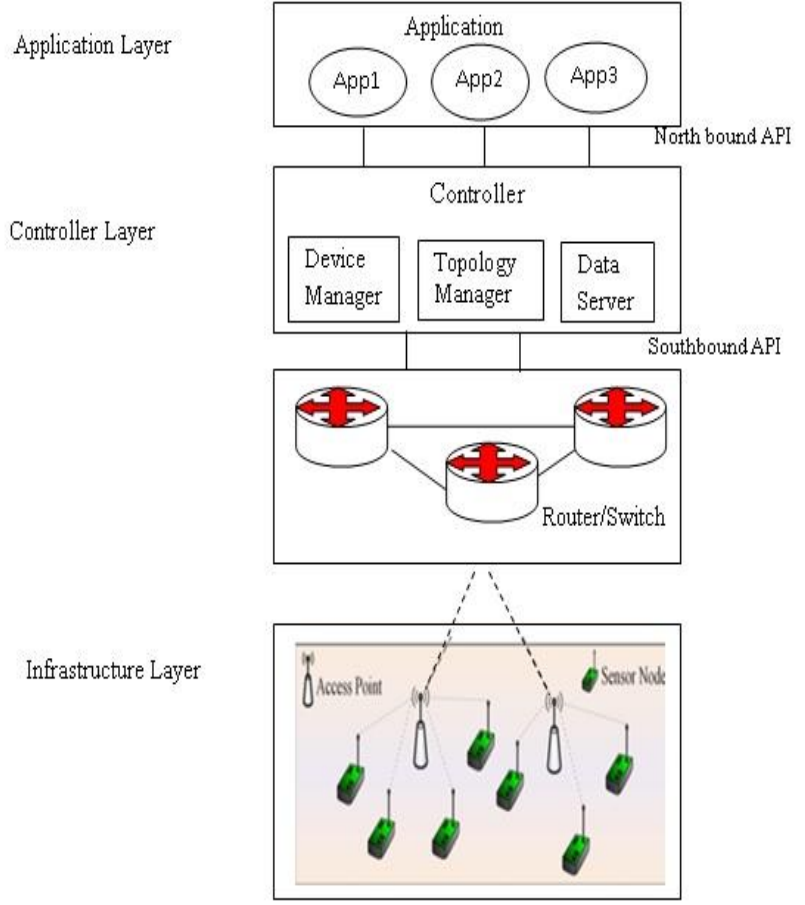

Fig 3 Architecture of SD-WSN

Fig. 3 shows architecture of SD-WSNs. The architecture is divided in to three layers. They are: i) Application Layer, ii) Control Layer, and iii) Infrastructure layer. The application layer of SD-WSNs contains various business applications to communicate with controller. The control layer separates data plane and control plane. The data plane for forwarding packets and control plane to make decision for routing the node. The infrastructure layer of SD-WSNs is a collection of sensor nodes which monitor and forward data in the wireless network. In WSN Flow-Sensor [21] adopt features of open flow controller. Flow sensor is responsible for forward packet in wireless network. Open flow allows controlling flow sensor and monitoring sensor traffic in WSN.

SDN-WISE [22] is software framework and prototype hardware for SD-WSN. SDNWISE is to simplify the management of nodes in wireless network. The Sink nodes are connected with network interface to forward the data from sensor node to the controller. The controller defines entire network behavior easily and continuously through the flow rules applied on sensor nodes. The WISE Flow table control by the controller for forwarding packets [23] [24].

Fig. 4 shows the structure of SDN-WISE protocol stack.

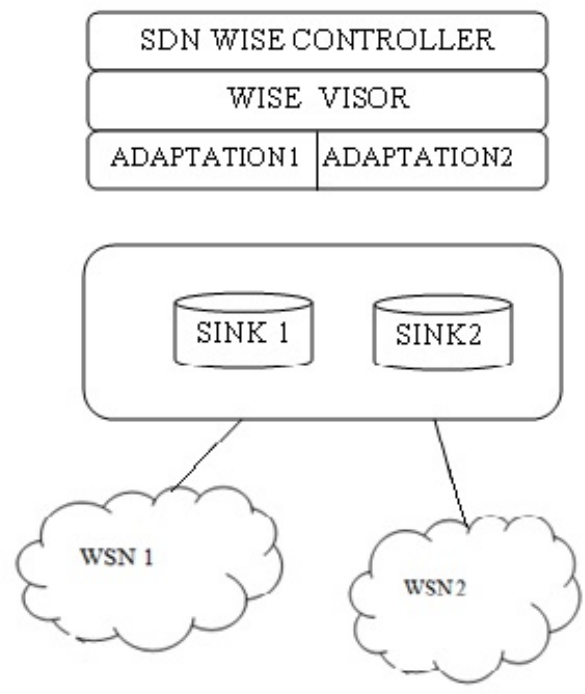

Fig 4 Protocol Stack SDN WISE

The WISE Visor is controlled by the controller for communication in WSN through the SINK node [25].

\section{LOAD BALANCING IN NETWORKING USING SDN}

In this paper proposed a work to make faultless continuous transmission and load balanced handover of destination node in WSN with SDN.Openflow protocol is not able to characterize the wireless sensor network and controller cannot manage the access point in infrastructure layer. The flow table updating is required for the future forwarding packet. The node in wsn is mobility so it is difficult to determine access point. The access point communicates with the controller to forward packet.

This new approach define two process

i) Propagation delay is to reduce by providing the flow table in advance to the controller.

ii) Calculate the weight of sink node to improve utilization of resources.

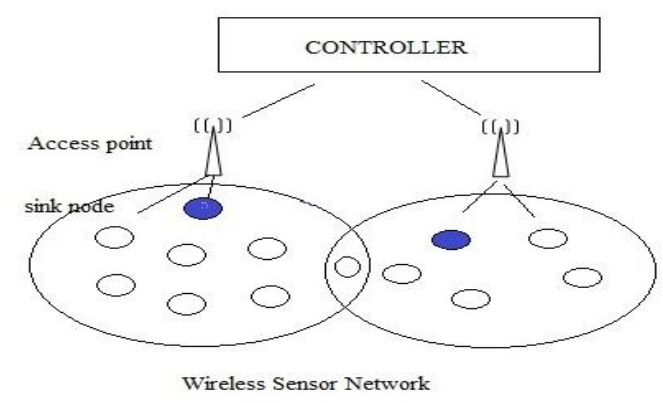

Fig 5 Load Balance in WSN 


\section{Sdn for Load Balancing Nodes in Wireless Sensor Network}

In Fig 5 Show that controller collects information from access point that makes rules for flow rules between nodes and sink node. The sink node gives information to access point through which it pass the flow table to the controller for making a decision.

The proposed sensor network provides information of flow table to the controller so that controller define rules for the node route decision making. The access point gives rules to the node for forwarding packet in network. The propagation delay reduces by providing flow table in advance to the controller. To improve the utilization of resources need to load balance in network. The weight of the sink node to be calculated based on strength of the signal and load of sink node. Load of sink node is traffic sent and received by the access point. Average weight of sink node is suitable for route decision. This makes increased utilization of resources in wireless sensor network.

\section{CONCLUSION}

Software Defined Networking is suitable for future networking. More research is based on routing algorithm for make a efficient utilize resources and minimize congestion due to overload balance.SDN with WSN makes efficient access of network resources, in future it will be suitable to change the software based network instead of hardware devices. SDN adoption can enforces network manageability, scalability, security in WSN.

\section{REFERENCES}

1. Zhaohui Chen, Zhaoyang Luo, Xiaohui Duan and Lianming Zhang, "Terminal hand over in software-defined WLANs", Journal on Wireless Communications and Networking,2020, https://doi.org/10.1186/s13638-020-01681

2. Deepak Singh Rana1, ShivAshish Dhondiyal, Sushil Kumar Chamoli, "Software Defined Networking (SDN) Challenges, issues and Solution", International Journal of Computer Sciences and Engineering, E-ISSN: 2347-2693 Vol.-7, Issue-1, Jan 2019.

3. Raphael Horvath, Dietmar Nedbala, Mark Stieningera ," A Literature Review on Challenges and Effects of Software Defined Networking”, science direct, Procedia Computer Science, Published by Elsevier, 64 ( 2015 ), pp $552-561$

4. Veeramani Shamugam, I Murray, J A Leong and Amandeep S Sidhu, "Software Defined Networking challenges and future direction: A case study of implementing SDN features on Open Stack private cloud", IOP Conf. Series: Materials Science and Engineering, March -2016, DOI: 10.1088/1757-899X/121/1/012003.

5. Habib Mustafa and Michael Menthe, "Software-Defined Wireless Sensor Networks: A Survey", Journal of Network and Computer Applications, June 2018.

6. H. I. Kobo, A. M. Abu-Mahfouz, G. P. Hancke, "A survey on software defined wireless sensor networks: Challenges and design requirements", IEEE 2017,pp 1872-1899.

7. Nam-Tuan Le, M. A. Hossain, Saha N, Md. Shareef Ifthekhar, Trang Nguyen, Chang Hyun Hong, Yeong Min Jang,'Software-Defined Networking Architecture for Energy Balance Sensor Network".

8. Junfeng Wang, Ping Zhai, Yin Zhang, Lei Shi1, Gaoxiang Wu, Xiaobo Shi, and Ping Zhou, "Software Defined Network Routing in Wireless Sensor Network", ICST Institute for Computer Sciences, Social Informatics and Telecommunications Engineering , pp. 3-11, 2018. https://doi.org/10.1007/978-3-319-69605-8_1

9. F. Hu and N. K. Sharma, "Security considerations in ad hoc sensornetworks,"AdHocNetworks,vol.3,no.1,pp.69-89,2005

10. J. Yick, B. Mukherjee, D. Ghosal, "Wireless sensor network survey", Comput. Netw. $52 \quad$ (12) (2008), 2292-2330. doi:10.1016/j.comnet.2008.04.002.

11. RenanC.A.Alves, DoriedsonA.G.Oliveira, GeovandroC.C.F.Pereira, BrunoC.Albertini, and CíntiaB.Margi, "Wireless Secure SDN-Based Communication for Sensor Networks", Hindawi Security and Communication Networks Volume 2018, Article ID 8734389, 14 pages https://doi.org/10.1155/2018/8734389
12. Caraguay ALV, Lopez LIB, Villalba LJG, "Evolution and Challenges of Software Defined Networking”, IEEE Communications Magazine, 2012.

13. Manar Jammala1, TaranpreetSingha, AbdallahShamia, RasoolAsalb, and YimingLic," Software-Defined Networking. State of the Art and Research Challenges, In Computer Networks”, vol. 72, pp. 74-98, 2014.

14. D. Kreutz, F. M. V. Ramos, P. E. Veríssimo, C. E. Rothenberg, S. Azodolmolky and S. Uhlig, "Software-Defined Networking: A Comprehensive Survey," in Proceedings of the IEEE, vol. 103, no. 1, pp. 14-76, Jan. 2015.

15. Ola Salman, Alichehab, Imad H.Elhajj, "SDN Controllers :A Comparative Study",April 2016,DOI: 10.1109/MELCON.2016.7495430.

16. Z.K.Khattak, M.Awais and A.iqbal,'Performance Evaluation of OpenDayLight SDN Controller",IEEE conference,2014.

17. la Salman, Imad H. Elhajj,Ali Chenab, "SDN controllers: A comparative study", 2016,DOI:10.1109/MELCON.2016.7495430

18. Abdelhadi Azzouni, Nguyen Thi Mai Trang, Raouf Boutaba, and Guy Pujolle," Limitations of Open Flow topology discovery protocol, In Ad Hoc Networking Workshop, 2017 16th Annual Mediterranean, pages 1-3. IEEE, 2017.

19. Erdal Akin, Turgay Korkmaz, "Comparison of Routing Algorithms with Static and Dynamic Link Cost in SDN", DOI: 10.1109/CCNC.2019.8651815.

20. J. Yick, B. Mukherjee, D. Ghosal," Wireless sensor network survey", Comput. Netw. $52 \quad$ (12) (2008) 2292-2330. doi:10.1016/j.comnet.2008.04.002.

21. A. Mahmud, R. Rahmani, "Exploitation of openflow in wireless sensor networks",2011 International Conference on Computer Science and Network Technology, Vol. 1, 2011, pp. 594-600. doi:10.1109/ICCSNT.2011.6182029.

22. L. Galluccio, S. Milardo, G. Morabito, S. Palazzo," Sdn-wise: Design, prototyping and experimentation of a stateful sdn solution for wireless sensor networks", 2015 IEEE Conference on Computer Communications (INFOCOM), 2015, pp. 513-521. doi:10.1109/INFOCOM.2015.7218418.

23. G. Gupta, M. Younis, "Load-balanced clustering of wireless sensor networks”, 2003. ICC'03. IEEE International Conference on, Vol. 3, IEEE, 2003, pp. 1848-1852.

24. S. Tomovic, I. Radusinovic, "Energy efficient target coverage in partially deployed software defined wireless sensor network", International Conference on Cognitive Radio Oriented Wireless Networks, Springer, 2016, pp. 729-740

25. Zhu,Z.Sun,Y.Lu,L.Zhang,Y.Wei,G. Min, “Centralized QoS routing using network calculus for SDN-based streaming media networks". IEEE Access.7,146566-146576(2019).

26. https://doi.org/10.1109/ACCESS.2019.2943518

\section{AUTHORS PROFILE}

D.Archana pursued Bachelor of Computer Application from Madras University in 2003 and Master of Computer Application from Sri Venkateswara University in 2007 and received M.Phil from Sri Chandrasekhandra Saraswathi Vishwa Mahavidyala University in 2015.Now she is pursuing Ph.D in the Research center of from Sri Chandrasekhandra Saraswathi Vishwa Mahavidyala University.Her main Research work focuses on SDN with Wireless Sensor Network. She is Currently working as Assistant Professor in the Department of Computer Science, Arcot Sri Mahalakshmi Women's College, Villapakkam,Ranipet,Since 2010.she has presented a paper on SDN with Cloud Computing in national conference and she is the author of 3 papers in international journals of repute.

Published By:

Blue Eyes Intelligence Engineering

\& Sciences Publication 


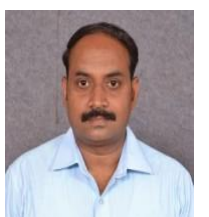

Dr.S.Prakasam received his B.Sc (Mathematics) and M.C.A form Madras University and he received his Ph.D from SCSVMV University. He has 19 years of teaching experience. He is currently serving as Associate Professor, Computer Science and Applications Department, Sri Chandrasekharendra Saraswathi Viswa Mahavidyalaya (SCSVMV) University, Enathur, Kanchipuram. His research interests include Knowledge Engineering, Data Communications. He has presented ten papers in international conference and he is the author of 55 papers in international journals of repute. He has guided 40 M.Phil., and 9 Ph.D(3 completed and currently 6 scholars doing the research under his guidance ).

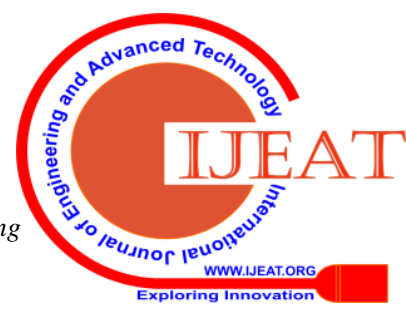

